\title{
Microfluidic Elaboration of Polymer Microfibers from Miscible Phases: Effect of Operating and Material Parameters on Fiber Diameter
}

Wasif Razzaq ${ }^{1,2}$, Christophe A. Serra, ${ }^{1 *}$ Leandro Jacomine ${ }^{1}$, Delphine Chan-Seng ${ }^{1}$

${ }^{1}$ Université de Strasbourg, CNRS, Institut Charles Sadron UPR 22, F-67000 Strasbourg, France Email: ca.serra@unistra.fr, +33 3.88.41.40.95

${ }^{2}$ Department of Materials Engineering, National Textile University, Sheikhupura Road, Faisalabad, 37610, Pakistan

Keywords: microfluidics, microfiber diameter, photopolymerization, capillary number

Background: fiber diameter is one of the most important morphological parameters which drives the applications of microfibers. This creates a need for the development of processes capable of producing a large variety of microfibers with a given diameter. To this regards, microfluidic spinning has recently emerged as an outstanding and simple technique for the production of micro- and nanofibers with controllable size and morphology.

Methods: herein, microfibers were produced from (macro)monomers or prepolymers (core phase) by in situ photoirradiation using a capillary-based microfluidic device and a miscible sheath phase of various viscosity. The effects of the flow rate of both phases as well as the viscosity of the sheath fluid, the capillary dimensions and the monomer volume fraction in core phase were thoroughly studied.

Significant findings: by calculating the capillary number ratio from the ratios of sheath to core flow rate and viscosity, an empirical relationship which perfectly predicts the microfiber diameter as a function of monomer volume fraction, the capillary number ratio and capillary inner diameter but independent of its outer diameter is extracted. This result paves the way to the continuousflow production of microfibers with well-controlled morphological characteristics. 


\section{Introduction}

Microfibers have attracted a lot of attention due to i) their large surface area to volume ratio, ii) their diverse properties arising from the great variety of materials they are made of, and iii) their ability to assemble into 3D complex structures and foldability.[1] These benefits enable polymer microfibers to have an excellent potential in many applications such as biomedicine,[2-6] fiber optics,[7] sensors,[8, 9] and water treatment.[10, 11] Different approaches have been employed to produce micro- and nanofibers such as melt spinning,[12] wet spinning,[13] draw spinning,[14] macromolecular assembly,[15] and electrospinning,[16] techniques relying on the physical mechanism of solidification to produce fibers, i.e. the starting raw material is a polymer solution. As such they suffer from limitations regarding i) the nature of the material employed and ii) the morphologies of fibers that can be achieved.[17] Hence, it is difficult to produce fibers with diverse morphologies and with a broad range of materials. Recently, microfluidic spinning has shown a great potential for the production of microfibers with diverse compositions, morphologies, and surface functionalities. This technique consists in stretching a stream of monomer or polymer solution (core phase) by an immiscible or miscible solution (continuous or sheath phase) inside a microfluidic device. Due to the small size of the device microchannel (ca. $100 \mu \mathrm{m})$, laminar flow is commonly achieved affording reproducibility and stability to the flow; two highly desirable features required for the production of fibers with given diameters and morphologies. By manipulating the flow inertia (i.e. individual solution flow rates), solution viscosity, interfacial tension between the core and sheath phases and taking possibly advantage of gravity forces, fibers with diverse morphologies such as grooved, flat, core-shell, hollow, and Janus can be easily produced. The choice of material becomes broader due to the panel of solidification methods, i.e. photopolymerization, ionic and chemical crosslinking, solvent exchange, and solvent evaporation. Fiber surface functionalization is also possible by encapsulation method or in situ chemistry.[1] Surface to volume ratio is one of the foremost fiber parameters that drive their applications. Since the latter is inversely proportional to the fiber diameter,[18] many properties such as 
mechanical,[19, 20] cell adhesion and proliferation,[20] biomimicking extracellular matrix,[21] optical extinction capacity,[22] and filtration performance[23] depend on the fiber diameter. Although, fiber diameter is a crucial parameter, the literature scarcely and partially reports on all the possible parameters responsible for controlling the fiber diameter in microfluidic spinning. Most reports address only the effect of sheath/continuous fluid and core/disperse fluids flow rates independently[24, 25] or in the form of sheath to core flow rate ratio (Qs/Qc) [26-29]. Liu et al. observed the effect of the capillary diameter on the fiber diameter using the thermoinitiated polymerization induced phase separation technique[30]. But there is no literature in which researchers interpreted these results into some mathematical form to predict the fiber diameters. Herein, we developed an empirical relationship which can predict the fiber diameter in relation with others operating and materials parameters for the case where the monomer and its polymer are miscible with the continuous phase. The investigation of operating parameters was not only limited to flow rates and capillary diameter, but the effect of viscosity of sheath fluid and monomer volume fraction in core phase was also investigated. Two monomers, tri(propylene glycol) diacrylate (TPGDA), poly(ethylene glycol) diacrylate (PEGDA), and one prepolymer, UV-curable adhesive NOA 89, were used as reference materials for the synthesis of fibers using in situ photoirradiation in a capillary-based microfluidic device. All results were used to extract an empirical correlation which predicted the final microfiber diameter upon variation of the capillary number ratio, monomer volume fraction, and internal capillary diameter.

\section{Results and Discussion}

The fibers were produced using a capillary-based microfluidic device (Fig. 1) involving two phases: 1) the core fluid (Фс) becoming the fiber upon photopolymerization and 2) the sheath fluid (Фs) consisting in poly(ethylene glycol) (PEG), whose flow rates are Qc and Qs respectively. PEG was used due to its miscibility with core fluid, commercial availability, high tunable 
viscosity with its molecular weight which prevents fast diffusion of Фc into Фs, and reasonable shearing force to produce thinner fibers. Фc comprised a diacrylate (TPGDA, PEGDA, or NOA 89) in ethanol in the presence of Irgacure 369 as photoinitiator.

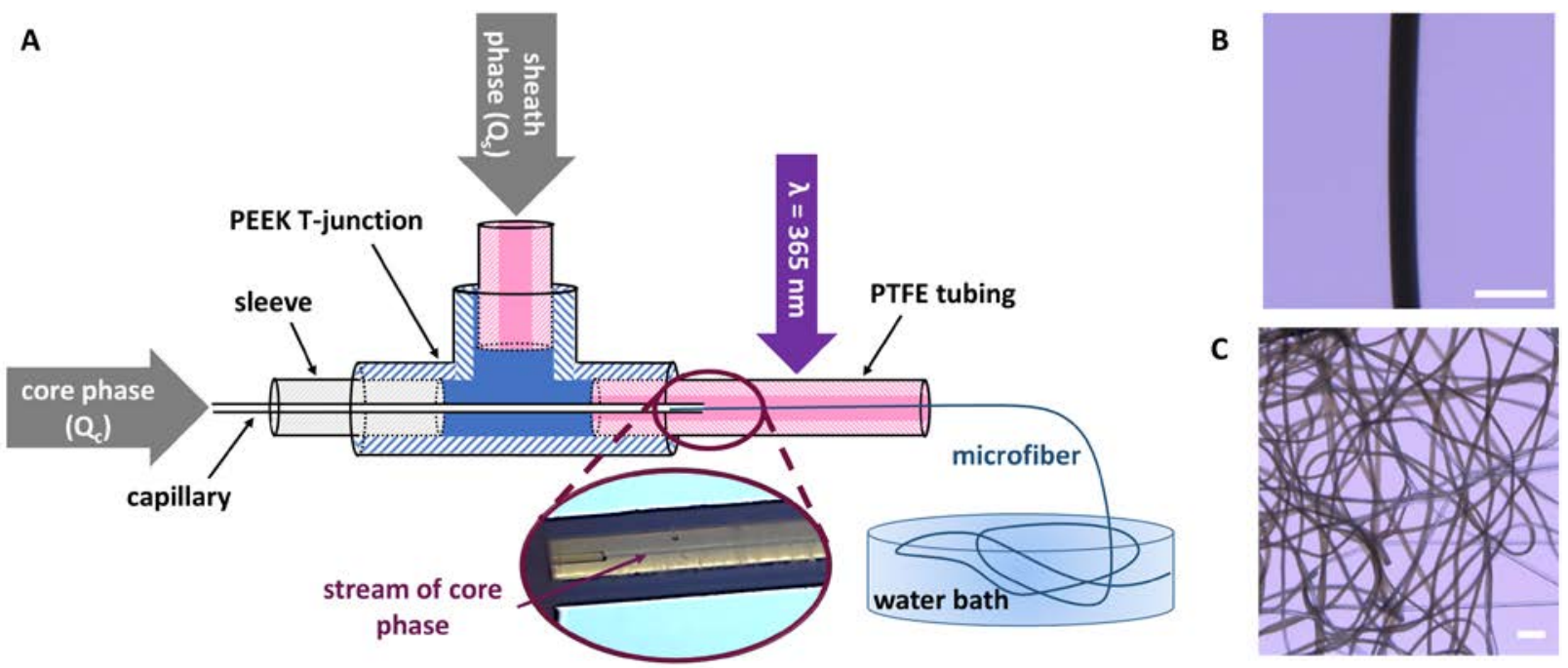

Fig. 1. (A) Capillary-based microfluidic device used to produce microfibers: Фs delivered from the top and Фc from the left of the T-junction using syringe pumps. The tip of the capillary, where Фs and Фc came into contact, has been magnified (inset). Numerical optical images of a single (B) and bundle $(\mathrm{C})$ of fibers (scale bar $=100 \mu \mathrm{m})$.

\subsection{Effect of the flow rate ratio $Q s / Q c$}

The effect of sheath and core flow rates on the fiber diameter was investigated showing a decrease of the fiber diameter as the flow rate of $\Phi$ s increased due to the increase in the shearing and stretching force exerted by $\Phi$ s over Фс. On the other hand, the diameter increased with an increase in the flow rate of $Ф \mathrm{c}$, because of the widening of the jet due to the increase in the volume of Фс injected.[31-34] To observe the overall impact of the flow rate on the fiber diameter, the effect of Qs/Qc on the fiber diameter instead of individual flow rates was studied using PEG 300 as Фs. In a first set of experiments, Qs was changed while keeping Qc constant to have different values of Qs/Qc (110, 150, 190, 230 and 280). In another set of experiments, the core flow rate was changed while keeping the sheath flow rate constant to produce the same values of Qs/Qc. The values of 
diameters produced in both set of experiments were similar (Fig. 2) meaning that the same Qs/Qc produced fibers of same diameters irrespective of the individual values of Qs or Qc. The reduction in diameter with the increase in Qs/Qc observed here has been also reported by other researchers.[35-37]

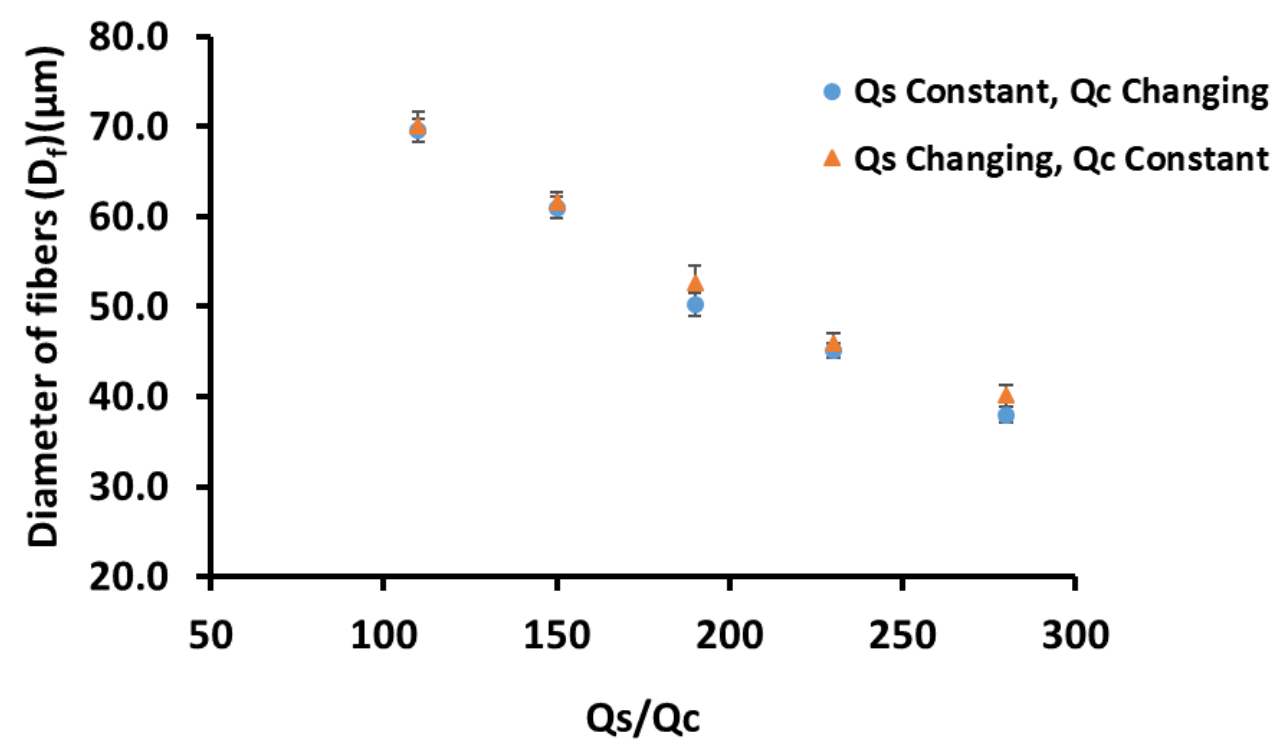

Fig. 2. Effect of Qs/Qc on the fiber diameter considering the effect of either the sheath flow rate (275 to $700 \mu \mathrm{L} / \mathrm{min}$ ) at $\mathrm{Qc}=2.5 \mu \mathrm{L} / \mathrm{min}$ ( $\triangle$ ) or the change core flow rate $(1.07$ to $2.72 \mu \mathrm{L} / \mathrm{min}$ ) at Qs $=300 \mu \mathrm{L} / \mathrm{min}(\bullet)$ producing the same Qs/Qc $(\mathrm{I} . \mathrm{D}=50 \mu \mathrm{m}$, outlet tubing internal diameter = $1.06 \mathrm{~mm}$, Фс = TPGDA/ethanol 60/40 v\%, Фs = PEG 300).

PEG of different molecular weights (PEG 200, PEG 400) and an equivolume mixture of PEG of different molecular weight (PEG 400/PEG 600) for Фs were studied (Fig. S1). The results of diameter variation with Qs/Qc for all the different $\Phi$ s are summarized in Fig. 3. Each curve showed the average diameter value of at least six replicates irrelevantly of the constant flow rate (Qs or Qc). The slopes of the curves were almost identical for each Фs indicating that Qs/Qc was a characteristic dimensionless parameter controlling the fiber diameter. 


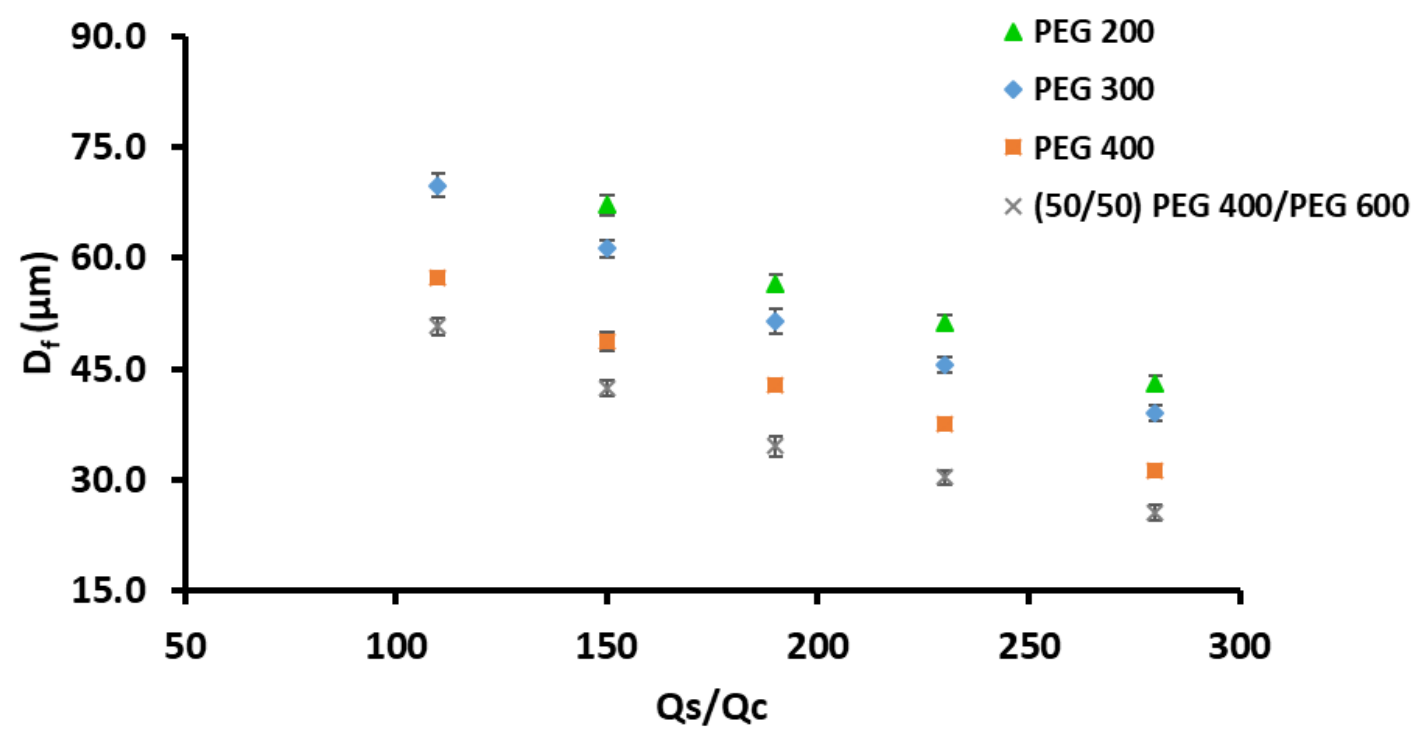

Fig. 3. Fiber diameter vs. Qs/Qc for different $\Phi$ s $\left(\mathrm{I} \cdot \mathrm{D}=50 \mu \mathrm{m}\right.$, O.D $=150 \mu \mathrm{m}$, $\Phi_{\mathrm{c}}=$ TPGDA/ethanol 60/40 v\%).

\subsection{Effect of viscosity ratio}

The effect of viscosity ratio on the fiber diameter was investigated. While keeping the viscosity of Фс constant (3.14 cP) for all the experiments, the viscosity of $\Phi$ s varied in the following order: PEG 400/PEG 600 (101.11 cP) > PEG 400 (82.11 cP) > PEG 300 (61.35 cP) > PEG 200 (42.03 cP). The fiber diameter was affected by both Qs/Qc and viscosity ratio $\left(\eta_{s} / \eta_{c}\right)$ (Fig. 4). Higher values of $\Phi$ s viscosity $\left(\eta_{\mathrm{s}}\right)$ produced fibers of lower diameter for the same Qs/Qc attributed to an increased shearing force induced by a more viscous $\Phi$ s resulting into the production of thinner core jet. Fig. 4 seemed to indicate that for a given $\Phi$ s, the fiber diameter followed a linear decrease with the increase in $\eta_{s} / \eta_{c}$. Hence, $\eta_{s} / \eta_{c}$ was also another dimensionless parameter that controlled the fiber diameter. 


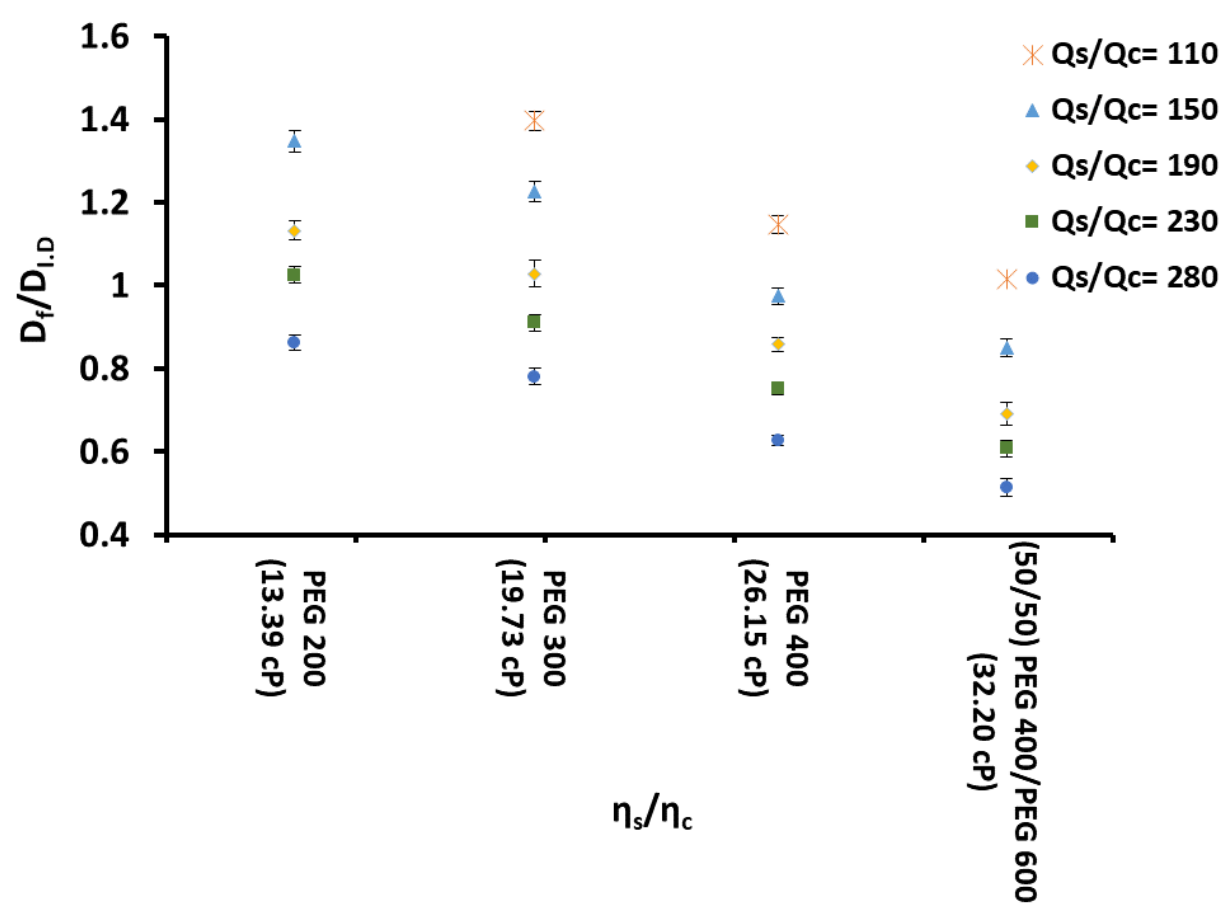

Fig. 4. Fiber diameter vs. sheath to core fluids viscosity ratio using PEG 200, PEG 300, PEG 400, PEG 400/PEG 600 as sheath fluids (I.D = $50 \mu \mathrm{m}$, O.D = $150 \mu \mathrm{m}$, Фс = TPGDA/ethanol 60/40 v\%).

\subsection{Capillary number ratio $\left(C a_{s} / C a_{c}\right)$}

Capillary number is defined as:

$$
\mathrm{Ca}=\eta \mathrm{v} / \gamma
$$

where $\eta$ and $\mathrm{v}$ are the viscosity and velocity of the fluid respectively, and $\gamma$ the interfacial tension between the fluids in contact. It compares the viscous force (proportional to the shear stress) to the interfacial force (proportional to the interfacial tension) and appears as an appropriate dimensionless number to account for the effects of the fluid velocity (or flow rate) and viscosity as stressed out in the above two previous studies. Two capillary numbers can be defined, $\mathrm{Ca}_{\mathrm{s}}=$ $\eta_{\mathrm{s}} \mathrm{v}_{\mathrm{s}} / \gamma$ and $\mathrm{Ca}_{\mathrm{c}}=\eta_{\mathrm{c}} \mathrm{v}_{\mathrm{c}} / \gamma$ for $\Phi$ s and $\Phi c$ respectively. For a given set of sheath and core fluids, the interfacial tension was the same and constant for both capillary numbers. Thus, one can define the capillary number ratio as:[38] 


$$
\frac{\mathrm{Ca}_{\mathrm{s}}}{\mathrm{Ca}_{\mathrm{c}}}=\frac{\eta_{\mathrm{s}} \cdot \mathrm{v}_{\mathrm{s}}}{\eta_{\mathrm{c}} \cdot \mathrm{v}_{\mathrm{c}}}
$$

This ratio can simultaneously accommodate the effects of $\eta_{s} / \eta_{c}$ and $Q_{s} / Q_{c}$. The latter can be expressed as a function of the velocity ratio $\left(\mathrm{v}_{\mathrm{s}} / \mathrm{v}_{\mathrm{c}}\right)$ since the inner cross sections of the capillary and outlet tubing were kept constant for all experiments. The sheath fluid velocity $\left(\mathrm{v}_{\mathrm{s}}\right)$ was evaluated by subtracting from the outlet tubing inner cross section the capillary outer cross section. To correlate all the data presented in the previous sections in the form of dimensionless numbers, the fiber diameter $\left(\mathrm{D}_{\mathrm{f}}\right)$ was arbitrarily normalized by the inner capillary diameter ( $\left.\mathrm{D}_{\mathrm{I} . \mathrm{D}}\right)$. After calculating the values of $\mathrm{v}_{\mathrm{s}} / \mathrm{v}_{\mathrm{c}}$ from the investigated $\mathrm{Q}_{\mathrm{s}} / \mathrm{Q}_{\mathrm{c}}$, the capillary ratios $\left(\mathrm{Ca}_{\mathrm{s}} / \mathrm{Ca}_{\mathrm{c}}\right)$ was accessed from Equation 2. The variations of the normalized fiber diameter $\left(\mathrm{D}_{\mathrm{f}} / \mathrm{D}_{\mathrm{I} . \mathrm{D}}\right)$ as a function of this capillary ratio (Fig. 5) was plotted for all the data presented in Fig. 4. All data fell down to a unique master curve highlighting the decrease of $D_{f}$ when the capillary number ratio was increased. The slight deviation for PEG 200 could be due to its lower viscosity allowing higher dilution of Фс into the bulk $\Phi$ s. This trend complied with the separate reduction in diameter observed with an increase of $\mathrm{Q}_{s} / \mathrm{Q}_{c}$ and $\eta_{\mathrm{s}} / \eta_{c}$ (Fig. 3 and 4 respectively). The same trend was reported for the production of polymer microparticle and oily droplets particles.[38, 39] 


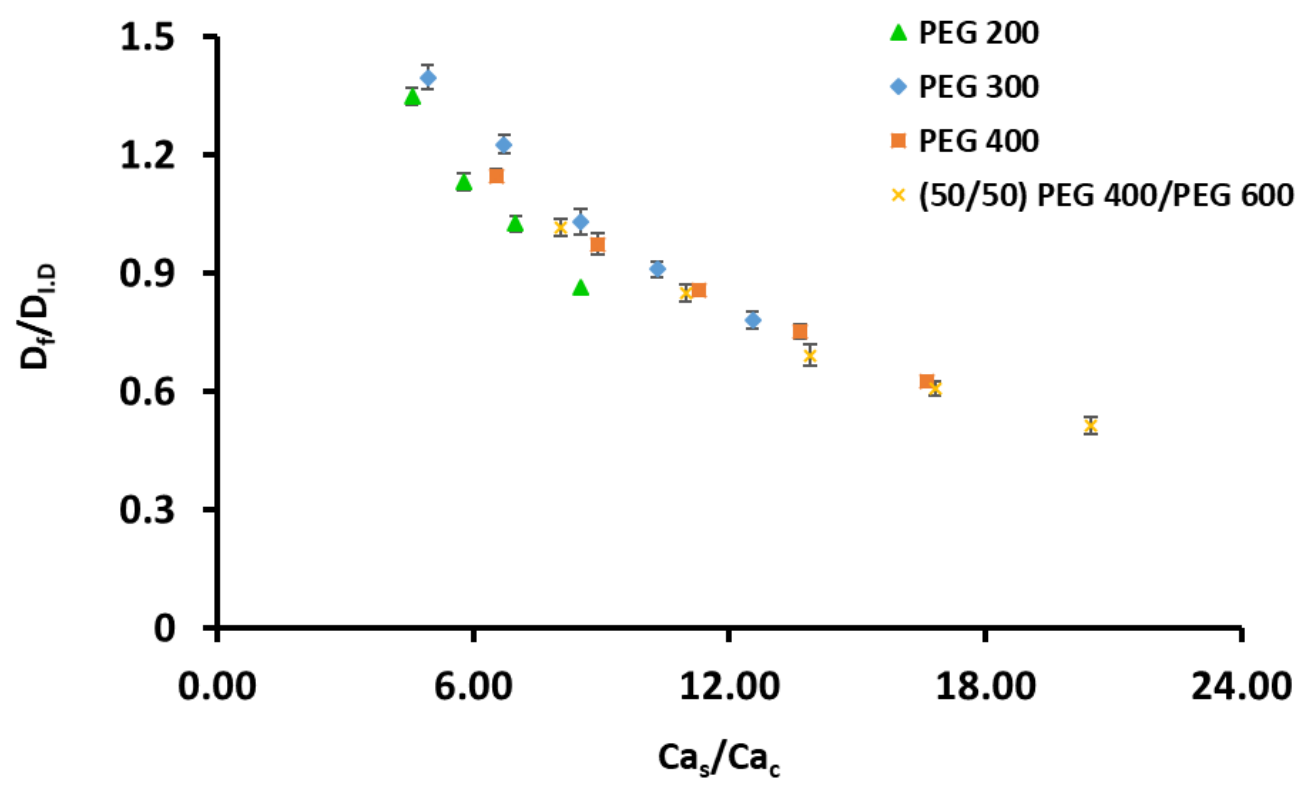

Fig. 5. Master curve presenting the variation of the diameter ratio $\left(D_{f} / D_{I . D}\right)$ with respect to the capillary number ratio $\left(\mathrm{Ca}_{\mathrm{s}} / \mathrm{Ca}_{\mathrm{c}}\right)$ according to the data from Fig. $3(\mathrm{I} . \mathrm{D}=50 \mu \mathrm{m}, \mathrm{O} . \mathrm{D}=150 \mu \mathrm{m}$, $\Phi \mathrm{c}=$ TPGDA/ethanol 60/40 v\%).

For the determination of a useful empirical correlation between dimensionless numbers allowing the prediction of $\mathrm{D}_{\mathrm{f}}$ as a function of operating and materials parameters, the data from Fig. $\mathbf{5}$ were represented in a logarithm plot (Fig. 6) (blue curve). From the linear variation of $\operatorname{Ln}\left(\mathrm{D}_{\mathrm{f}} / \mathrm{D}_{\mathrm{I.D}}\right)$ with $\mathrm{Ln}\left(\mathrm{Ca}_{\mathrm{s}} / \mathrm{Ca}_{\mathrm{c}}\right)$, the following correlation was extracted by linear regression:

$$
\frac{D_{f}}{D_{I . D}}=K_{0}\left(\frac{C a_{s}}{C a_{c}}\right)^{-0.63}
$$

with $\mathrm{K}_{0}=3.71$.

Positive values of $\operatorname{Ln}\left(\mathrm{D}_{\mathrm{f}} / \mathrm{D}_{\text {I.D }}\right)$ in Fig. 6 indicated fibers which diameters were greater than the inner capillary diameter while the negative values indicated smaller diameters. It was observed that a wide range of diameters, smaller or greater than the size of capillary diameter can be obtained. In the above-mentioned experiments, $\mathrm{K}_{0}$ is a constant but could possibly vary with the capillary dimensions. 


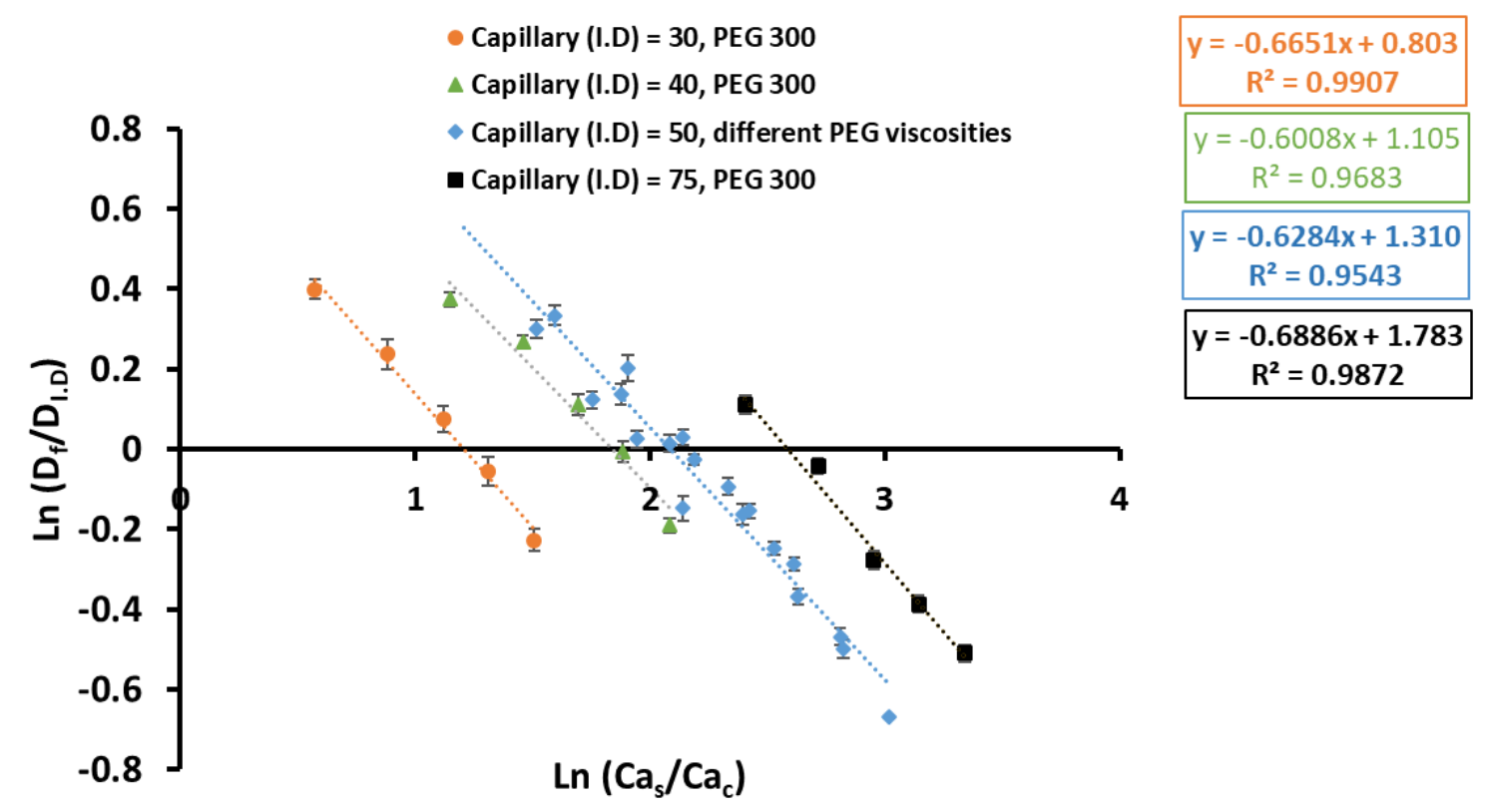

Fig. 6. Ln-Ln graph of fiber diameter variation with respect to $\mathrm{Ca}_{s} / \mathrm{Ca}_{c}$ for the experimental data of Fig. 5 (blue curve) (I.D = 30, 40, 50, and $75 \mu \mathrm{m}$, O.D = $150 \mu \mathrm{m})$. The dotted line indicates the linear regression fit (Equation 3).

\subsection{Effect of outer and inner capillary diameter}

The effect of the outer capillary diameter (O.D) on $\mathrm{D}_{\mathrm{f}}$ was assessed using O.D of 150, 190, 280 and $365 \mu \mathrm{m}$, while keeping the inner diameter (I.D) equal to $50 \mu \mathrm{m}$ (Qc $=2.5 \mu \mathrm{L} / \mathrm{min}$, while varying Qs from 275 to $700 \mu \mathrm{L} / \mathrm{min}$ ). The logarithm of $\mathrm{D}_{\mathrm{f}}$ followed a linear variation with respect to the logarithm of Qs/Qc irrelevantly of O.D (Fig. S2). Furthermore, the linear regression equation was the same as the one found for the blue curve (I.D $=50 \mu \mathrm{m}$, Fig. 6). It was concluded that the capillary outer diameter did not affect $\mathrm{D}_{\mathrm{f}}$ in the size range investigated. Inversely to the production of droplets, whose diameter is fixed when they detach from the capillary tip,[38] fiber diameter is mainly set downstream to the capillary tip when the shear stresses of $\Phi$ s and Фc equilibrate. The effect of the O.D was irrelevant.

Similarly, the effect of I.D was investigated using capillaries with the same O.D (150 $\mu \mathrm{m})$ but different I.D (30, 40, 50 and $75 \mu \mathrm{m}$ ) with the same conditions for Qc and Qs as the previous study 
conducted with a constant I.D. Varying I.D resulted in a set of parallel lines (same slopes around -0.63) but exhibited different y-intercepts as a function of I.D (Fig. 6) indicating that I.D played a role on the resulting $\mathrm{D}_{\mathrm{f}}$ through the $\mathrm{K}_{0}$ parameter of Equation 3. To assess more precisely this dependence, the different y-intercepts (i.e. $\operatorname{Ln}\left(\mathrm{K}_{0}\right)$ ) values extracted from Fig. 6 were plotted as a function of $\operatorname{Ln}\left(D_{\text {I.D }}\right)$ (Fig. 7) showing a linear dependence between $\operatorname{Ln}\left(K_{0}\right)$ and $\operatorname{Ln}\left(\mathrm{D}_{\text {I.D }}\right)$. This dependence can be expressed by the following equation:

$$
\operatorname{Ln}\left(K_{0}\right)=1.06 \operatorname{Ln}\left(D_{I D}\right)-2.82
$$

which can be rewritten as:

$$
K_{0}=\exp ^{-2.82} D_{I D}^{1.06}
$$

Introducing $K_{0}$ into Equation 3 and taking the average value of the slopes in Fig. 6 (-0.65) gave:

$$
\frac{D_{f}}{D_{I . D}}=K_{1} D_{I . D}^{1.06}\left(\frac{C a_{s}}{C a_{c}}\right)^{-0.65}
$$

with $K_{1}=0.06 \mu \mathrm{m}^{-1}$.

Extracting $\mathrm{D}_{\mathrm{f}}$ from above equation resulted in:

$$
D_{f}=K_{1} D_{I . D}^{2.06}\left(\frac{C a_{s}}{C a_{c}}\right)^{-0.65}
$$

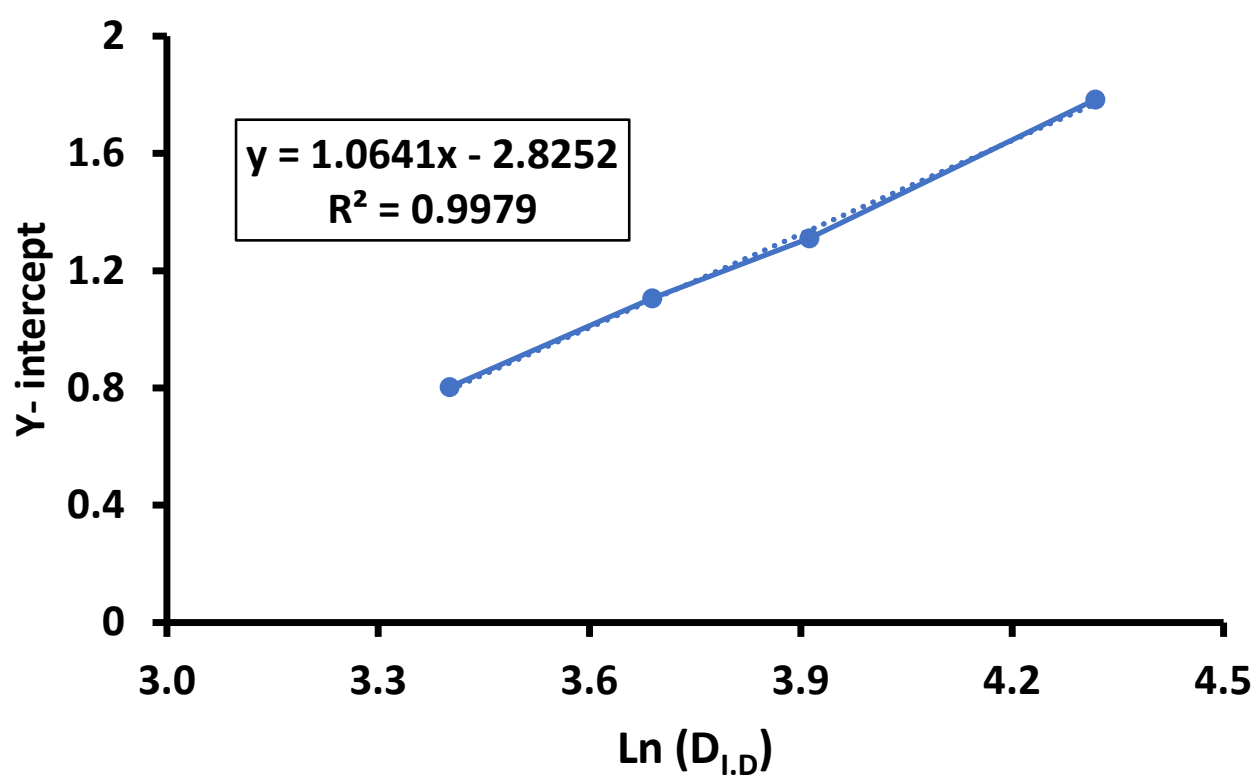

Fig. 7. Variation of the y-intercept (Fig. 6) with respect to logarithm of I.D (O.D = $150 \mu \mathrm{m})$. 


\subsection{Effect of monomer volume fraction}

The effect of Фc composition was investigated by varying the monomer volume fraction $\left(\mathrm{X}_{\mathrm{v}, \mathrm{m}}\right.$, Equation 8) from 10 to $80 \%$.

$$
X_{v, m}=\frac{V_{m}}{V_{m}+V_{s}}
$$

where $V_{m}$ and $V_{s}$ are the monomer and solvent (ethanol) volumes in core phase respectiely The logarithm of $\mathrm{D}_{\mathrm{f}}$ followed a linear increase with respect to the logarithm of the volume fraction (Fig. 8) which was expressed as:

$$
D_{f}=K_{2} X_{v, m}^{0.47}
$$

with $K_{2}=66 \mu \mathrm{m}$.

As the monomer volume fraction increased, a larger portion of the monomer core phase volume was polymerized resulting in a thicker fiber. Experiments with $\mathrm{X}_{\mathrm{v}, \mathrm{m}}$ above $80 \%$ led to clogging due to the buckling effect resulting from the fast polymerization induced by the high concentration of monomer.

By replacing $K_{1}$ (Equation 7) by the right-hand side expression of Equation 9, the overall correlation could be expressed as:

$$
D_{f}=K_{3} X_{v, m}^{0.47} D_{I . D}^{2.06}\left(\frac{C a_{s}}{C a_{c}}\right)^{-0.65}
$$

$K_{3}$ was determined to be equal to $0.12 \mu \mathrm{m}^{-1}$ by minimizing the mean squared error between all experimental $D_{\mathrm{f}}$ measured and the fiber diameters returned by Equation 10 under same experimental conditions (Fig. S3). Equation 10 represented an empirical correlation allowing a fairly well prediction of $\mathrm{D}_{\mathrm{f}}$, in the range of $\mathrm{Ca}_{\mathrm{s}} / \mathrm{Ca}_{\mathrm{c}}$ between 25 and 84 , as a function of operating parameters, i.e. I.D, individual capillary number of $\Phi$ s and Фс and monomer volume fraction in Фc (Fig. S3). 


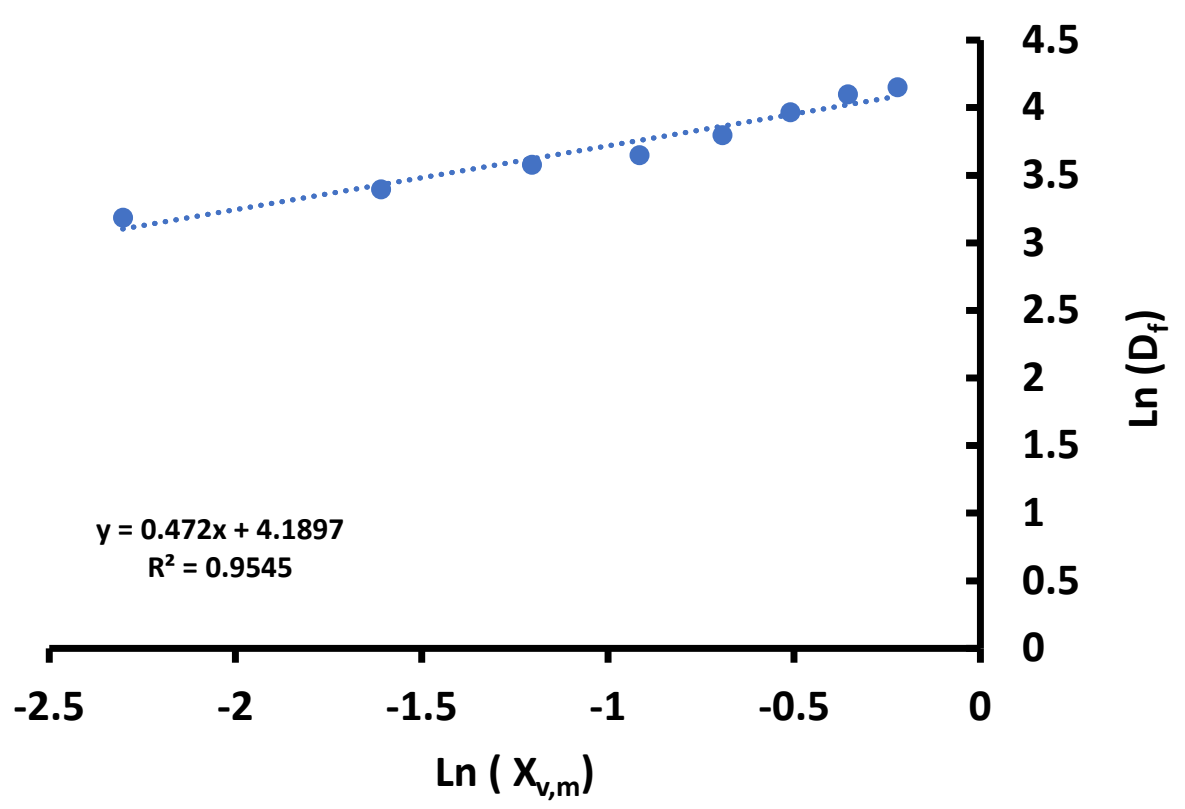

Fig. 8. Variation of the fiber diameter with respect to the monomer volume fraction (I.D $=50 \mu \mathrm{m}$, O.D $=150 \mu \mathrm{m}$, Фс = TPGDA/ethanol, $\Phi \mathrm{s}=$ PEG 300).

\subsection{Effect of material system}

Equation 10 was obtained with one specific material system, i.e. TPGDA as Фc and PEG as Фs.

To check the relevancy of this empirical relationship, two additional Фс were investigated: PEGDA with a molecular weight of $400 \mathrm{~g} / \mathrm{mol}$ and NOA 89 while keeping the same Фs (PEG 300). It was observed that if the slope remained the same irrelevantly of the material system studied, $K_{0}$ value (and subsequent $K_{3}$ ) changed noticeably (Table 1). The change was moderate for TPGDA and NOA 89 while it was much more pronounced for PEGDA. This must be related to the core phase viscosity ( $\eta_{c}$, Table 1) which is twice higher for PEGDA than for the other two core phases investigated. Thus, one can make the hypothesis that PEGDA monomer diffuses less in the sheath phase. As a result, $\mathrm{D}_{\mathrm{f}}$ will be thinner which will be expressed by a lower diameter and thus a lower y-intercept. 
Table 1. Values of the slope, y-intercept for $\operatorname{Ln}\left(\mathrm{D}_{\mathrm{f}} / \mathrm{D}_{\text {I.D. }}\right)$ vs. $\mathrm{Ln}\left(\mathrm{Ca}_{\mathrm{s}} / \mathrm{Ca}_{\mathrm{c}}\right)$ plots (Fig. S4) and viscosity using different core phases (I.D and O.D of $50 \mu \mathrm{m}$ and $150 \mu \mathrm{m}$ respectively).

\begin{tabular}{ccccc}
\hline Core phase & Slope & $\mathbf{y}$-intercept & $\mathbf{K}_{\mathbf{0}}\left(\boldsymbol{\mu \mathbf { m } ^ { - 1 } )}\right.$ & $\mathbf{\eta}_{\mathbf{s}}(\mathbf{c P})$ \\
\hline TPGDA & -0.628 & 1.31 & 3.71 & 3.14 \\
NOA 89 & -0.688 & 1.24 & 3.46 & 3.5 \\
PEGDA & -0.622 & 0.80 & 2.23 & 7.04 \\
\hline
\end{tabular}

\subsection{Monodispersity and reproducibility of fiber diameter}

The robustness of the process was proved by evaluating the monodispersity and reproducibility of $\mathrm{D}_{\mathrm{f}}$. The coefficient of variation $(\mathrm{CV})$, defined as the ratio between the standard deviation $\sigma$ (Equation 12) of all the diameters measured and the average fiber diameter $\mathrm{D}_{\mathrm{f}, \mathrm{avg}}$, was evaluated (Fig. S5):

$$
C V=\sigma / D_{f, a v g}
$$

$\mathrm{CV}$ is generally reported to check against the monodispersity of micron-sized droplets or particles. It is commonly admitted that CVs below 5\% are the signature of monodisperse objects. It was extended to fibers and the monodispersity was evaluated by measuring the diameter at different random locations along the fiber length for different sheath and core phase flow rates and for a capillary with I.D = $50 \mu \mathrm{m}$ and O.D = $150 \mu \mathrm{m}$. It remained below 5\% in all situations indicating that the developed process allowed producing monodisperse fibers (Fig. S5a).

As for the reproducibility, it was evaluated by determining the average diameter of fibers obtained for three batches with the same operating and material parameters carried out on different days. The results (Fig. S5b) indicated that whatever the sheath phase employed, the returned CVs were always lower than 5\% highlighting the strong reproducibility of the process. 


\section{Conclusion}

Polymer microfibers of two different monomers and one photocurable adhesive with diameters as low as $23 \mu \mathrm{m}$ were produced by in situ photopolymerization using a capillary-based coaxial microfluidic device and a miscible PEG sheath fluid. The impact of different operating and material parameters such as volume fraction of monomer in core phase, flow rate ratio of sheath to core fluid, viscosity of the sheath fluid and dimensions of the capillary on the resulting fiber's diameter were studied. The diameter of the fiber was inversely related to the flow rate ratio and viscosity of the sheath fluid. The size of the fiber could also be varied by changing the inner diameter of the capillary but was not affected by the outer diameter. From all the data collected, an empirical relationship which precisely predicted the fiber diameter as a function of the capillary’s inner diameter, monomer volume fraction and capillary number ratio of sheath to core fluid was successfully extracted. Furthermore, the coefficient of variation of the fibers' diameter was consistently less than $5 \%$ thus highlighting a robust and reproducible process for the production of monodisperse fibers.

\section{Acknowledgments}

Authors thank W. Drenckhan for discussions and A. Collard for all technical aspects. Authors are grateful to P. Allgayer for mechanical engineering support. WR would like to acknowledge the Higher Education Commission of Pakistan (HEC) for his Ph.D. fellowship.

\section{References}

[1] X.-Y. Du, Q. Li, G. Wu, S. Chen, Multifunctional micro/nanoscale fibers based on microfluidic spinning technology, Adv. Mater. 31 (2019) 1903733.

[2] M. Angelozzi, M. Miotto, L. Penolazzi, S. Mazzitelli, T. Keane, S.F. Badylak, et al., Composite ECMalginate microfibers produced by microfluidics as scaffolds with biomineralization potential, Mater. Sci. Eng. C 56 (2015) 141-153. 
[3] T. Takei, N. Kishihara, S. Sakai, K. Kawakami, Novel technique to control inner and outer diameter of calcium-alginate hydrogel hollow microfibers, and immobilization of mammalian cells, Biochem. Eng. J. 49 (2010) 143-147.

[4] Y.-S. Lin, K.-S. Huang, C.-H. Yang, C.-Y. Wang, Y.-S. Yang, H.-C. Hsu, et al., Microfluidic synthesis of microfibers for magnetic-responsive controlled drug release and cell culture, PLoS One 7 (2012) e33184.

[5] C. Haynl, E. Hofmann, K. Pawar, S. Förster, T. Scheibel, Microfluidics-produced collagen fibers show extraordinary mechanical properties, Nano Lett. 16 (2016) 5917-5922.

[6] T. Sun, Q. Huang, Q. Shi, H. Wang, C. Hu, P. Li, et al., Assembly of alginate microfibers to form a helical structure using micromanipulation with a magnetic field, J. Micromech. Microeng. 26 (2016) 105017.

[7] R. Blue, D. Uttamchandani, Recent advances in optical fiber devices for microfluidics integration, J. Biophotonics 9 (2016) 13-25.

[8] K. Liu, Z. Zhou, X. Yan, X. Meng, H. Tang, K. Qu, et al., Polyaniline nanofiber wrapped fabric for high performance flexible pressure sensors, Polymers 11 (2019) 1120.

[9] M. Yang, J. Pan, A. Xu, L. Luo, D. Cheng, G. Cai, et al., Conductive cotton fabrics for motion sensing and heating applications, Polymers 10 (2018) 568.

[10] E. Korina, O. Stoilova, N. Manolova, I. Rashkov, Polymer fibers with magnetic core decorated with titanium dioxide prospective for photocatalytic water treatment, J. Environ. Chem. Eng. 6 (2018) 20752084.

[11] L. Jurecska, K. Barkács, É. Kiss, G. Gyulai, T. Felföldi, B. Törő, et al., Intensification of wastewater treatment with polymer fiber-based biofilm carriers, Microchem. J. 107 (2013) 108-114.

[12] L. Cui, N. Zhang, W. Cui, P. Zhang, X. Chen, A Novel Nano/Micro-Fibrous Scaffold by MeltSpinning Method for Bone Tissue Engineering, Journal of Bionic Engineering 12 (2015) 117-128.

[13] O. Nechyporchuk, T. Yang Nilsson, H. Ulmefors, T. Köhnke, Wet Spinning of Chitosan Fibers: Effect of Sodium Dodecyl Sulfate Adsorption and Enhanced Dope Temperature, ACS Applied Polymer Materials 2 (2020) 3867-3875. 
[14] S. Liao, X. Bai, J. Song, Q. Zhang, J. Ren, Y. Zhao, et al., Draw-Spinning of Kilometer-Long and Highly Stretchable Polymer Submicrometer Fibers, Advanced Science 4 (2017) 1600480.

[15] Y. Loo, S. Zhang, C.A.E. Hauser, From short peptides to nanofibers to macromolecular assemblies in biomedicine, Biotechnology Advances 30 (2012) 593-603.

[16] J. Lin, B. Ding, J. Yu, Y. Hsieh, Direct Fabrication of Highly Nanoporous Polystyrene Fibers via Electrospinning, ACS Applied Materials \& Interfaces 2 (2010) 521-528.

[17] A. Duboin, R. Middleton, F. Malloggi, F. Monti, P. Tabeling, Cusps, spouts and microfiber synthesis with microfluidics, Soft Matter 9 (2013) 3041-3049.

[18] K. Singha, S. Maity, M. Singha, P. Paul, D.P. Gon, Effects of fiber diameter distribution of nonwoven fabrics on its properties, Int. J. Textile Sci. 1 (2012) 7-14.

[19] K. Young, F.M. Blighe, J.J. Vilatela, A.H. Windle, I.A. Kinloch, L. Deng, et al., Strong dependence of mechanical properties on fiber diameter for polymer-nanotube composite fibers: Differentiating defect from orientation effects, ACS Nano 4 (2010) 6989-6997.

[20] H.H. Kim, M.J. Kim, S.J. Ryu, C.S. Ki, Y.H. Park, Effect of fiber diameter on surface morphology, mechanical property, and cell behavior of electrospun poly(e-caprolactone) mat, Fibers Polym. 17 (2016) 1033-1042.

[21] M. Chen, P.K. Patra, S.B. Warner, S. Bhowmick, Role of fiber diameter in adhesion and proliferation of NIH 3T3 fibroblast on electrospun polycaprolactone scaffolds, Tissue Eng. 13 (2007) 579-587.

[22] J. Yang, F. He, H. Wu, Y. Liang, Y. Wang, Z. Sun, Engineering surface and optical properties of $\mathrm{TiO}_{2}$-coated electrospun PVDF nanofibers via controllable self-assembly, Nanomater. 8 (2018) 741.

[23] S. Chang, A.G. Fane, The effect of fibre diameter on filtration and flux distribution — relevance to submerged hollow fibre modules, J. Membr. Sci. 184 (2001) 221-231.

[24] W. Jeong, J. Kim, S. Kim, S. Lee, G. Mensing, D.J. Beebe, Hydrodynamic microfabrication via"on the fly" photopolymerization of microscale fibers and tubes, Lab Chip 4 (2004) 576-80.

[25] M. Hu, R. Deng, K.M. Schumacher, M. Kurisawa, H. Ye, K. Purnamawati, et al., Hydrodynamic spinning of hydrogel fibers, Biomaterials 31 (2010) 863-9.

[26] A.L. Thangawng, P.B. Howell, Jr., C.M. Spillmann, J. Naciri, F.S. Ligler, UV polymerization of hydrodynamically shaped fibers, Lab Chip 11 (2011) 1157-60. 
[27] S. Nakajima, R. Kawano, H. Onoe, Stimuli-responsive hydrogel microfibers with controlled anisotropic shrinkage and cross-sectional geometries, Soft Matter 13 (2017) 3710-3719.

[28] A.L. Thangawng, P.B. Howell, Jr., J.J. Richards, J.S. Erickson, F.S. Ligler, A simple sheath-flow microfluidic device for micro/nanomanufacturing: fabrication of hydrodynamically shaped polymer fibers, Lab Chip 9 (2009) 3126-30.

[29] A. Venkateshwarlu, R.P. Bharti, Effects of capillary number and flow rates on the hydrodynamics of droplet generation in two-phase cross-flow microfluidic systems, Journal of Taiwan Institute of Chemical Engineers 129 (2021) 64-79.

[30] W. Liu, Z. Xu, L. Sun, P. Guo, C. Zeng, C. Wang, et al., Polymerization-induced phase separation fabrication: A versatile microfluidic technique to prepare microfibers with various cross sectional shapes and structures, Chemical Engineering Journal 315 (2017) 25-34.

[31] C.M. Hwang, A. Khademhosseini, Y. Park, K. Sun, S.-H. Lee, Microfluidic chip-based fabrication of PLGA microfiber scaffolds for tissue engineering, Langmuir 24 (2008) 6845-6851.

[32] F. Wu, X.-J. Ju, X.-H. He, M.-Y. Jiang, W. Wang, Z. Liu, et al., A novel synthetic microfiber with controllable size for cell encapsulation and culture, J. Mater. Chem. B 4 (2016) 2455-2465.

[33] B.K. Pullagura, V. Gundabala, Microfluidics-based on-demand generation of nonwoven and single polymer microfibers, Langmuir 36 (2020) 1227-1234.

[34] M. Zhou, J. Gong, J. Ma, Continuous fabrication of near-infrared light responsive bilayer hydrogel fibers based on microfluidic spinning, e-polym. 19 (2019) 215-224.

[35] Z. Bai, J.M. Mendoza Reyes, R. Montazami, N. Hashemi, On-chip development of hydrogel microfibers from round to square/ribbon shape, M. Mater. Chem. A 2 (2014) 4878-4884.

[36] M. Nie, S. Takeuchi, Microfluidics based synthesis of coiled hydrogel microfibers with flexible shape and dimension control, Sens. Actuators, B 246 (2017) 358-362.

[37] A. Gursoy, K. Iranshahi, K. Wei, A. Tello, E. Armagan, L.F. Boesel, et al., Facile fabrication of microfluidic chips for 3D hydrodynamic focusing and wet spinning of polymeric fibers, Polymers 12 (2020) 633. 
[38] C. Serra, N. Berton, M. Bouquey, L. Prat, G. Hadziioannou, A predictive approach of the influence of the operating parameters on the size of polymer particles synthesized in a simplified microfluidic system, Langmuir 23 (2007) 7745-7750.

[39] E. Chiarello, L. Derzsi, M. Pierno, G. Mistura, E. Piccin, Generation of oil droplets in a non-newtonian liquid using a microfluidic T-junction, Micromachines 6 (2015) 1825-1835. 